\title{
Expression of Chikungunya virus-like particles
}

\author{
R. A. Velez*, A. P. Alves de Matos**, R. Parreira*, J. Piedade, B. Matos***, C. Correia*** and A. \\ Esteves* \\ * Grupo de Virologia, Unidade de Microbiologia Médica, UPMM, Instituto de Higiene e Medicina \\ Tropical, UNA, 1349-008 Lisboa, Portugal \\ ** Centro Hospitalar de Lisboa Central - HCC, Serviço de Anatomia Patológica, R. da Beneficência \\ 8, 1069-166 Lisboa and Centro de Estudos do Ambiente e do Mar (CESAM) - Faculdade de Ciências \\ da Universidade de Lisboa, Campo Grande, 1149-016, Lisboa, Portugal \\ *** Centro Hospitalar de Lisboa Central - HCC, Serviço de Anatomia Patológica, R. da Beneficência \\ 8, 1069-166 Lisboa, Portugal
}

Chikungunya virus (CHIKV) is a mosquito-transmitted alphavirus responsible for painful disabling polyarthritis, undergoing re-emergence in the Indian Ocean and the Asian continent[1]. Travelling and changing patterns of vector distribution, and abundance due to climate changes, make CHIKV a global threat without effective control strategies. One approach to reduce the CHIKV burden is the development of a vaccine. Virus-like particles (VLPs) are a safe and highly effective class of subunit vaccines that mimic the overall structure of virus particles. Accordingly, we aim to produce CHIKV VLPs in a baculovirus-insect cell system, well known for allowing high yields of recombinant protein expression, to be used as a vaccine preparation.

The ORF coding the CHIKV structural proteins is $3.8 \mathrm{~kb}$ long, and therefore there is a chance for generating recombinant baculoviruses with spurious stop codons leading to truncated polyproteins. To minimize this possibility, the CHIKV structural sequence was amplified by RT-PCR as a SacI-NotI fragment, and cloned into the mammalian expression vector pLEXm (a gift from R. Aricescu, Oxford, UK) downstream the strong chick beta actin promoter. Several clones with the correct insert size were obtained and transfected into HEK293T cells using polyethylenimine as the transfection reagent[2]. Five recombinant vectors were shown to express CHIKV proteins by immunofluorescence (IFA) and Western blotting (WB) analyses using a polyclonal serum against CHIKV (Figure 1A). One clone, with high levels of IFA labelling, coded for a truncated viral polyprotein (WB data), while three others, with a lower IFA signal, originated low levels of viral envelope glycoproteins correctly processed. Cells transfected with clone p67 showed IFA staining and viral glycoprotein pattern on WB similar to cells infected with CHIKV (Figure 1A compare lanes I and p67). Precipitation of clarified cell culture medium from p67 transfected cells, and from CHIKV infected cells, with PEG 8000 generated pellets with an identical content of viral glycoproteins on WB (Figure 1B). These results strongly indicate that p67 transfected cells express CHIKV structural proteins that are assembled into VLPs. Transmission electron microscopy analysis of p67 transfected cells (Figure 3) confirmed the assembly of VLPs with a morphology similar to the virus particles produced in CHIKV infected cells (Figure 2). Quite unexpectedly, transfected cells expressing only the CHIKV structural proteins (Figure 3) exhibit cytoplasmic membrane rearrangements similar to the vesicle arrays observed in CHIKV infected cells (Figure 2) and reported as induced by non-structural viral proteins. In conclusion, p67 will be used as a source of CHIKV structural genes for construction of recombinant baculoviruses.

\section{References}

1. Townson H. and Nathan M.B., Trans R Soc Trp Med Hyg 102: 308-309, 2008

2. Aricescu et al., Acta Cryst. D62: 1243-1250, 2006 


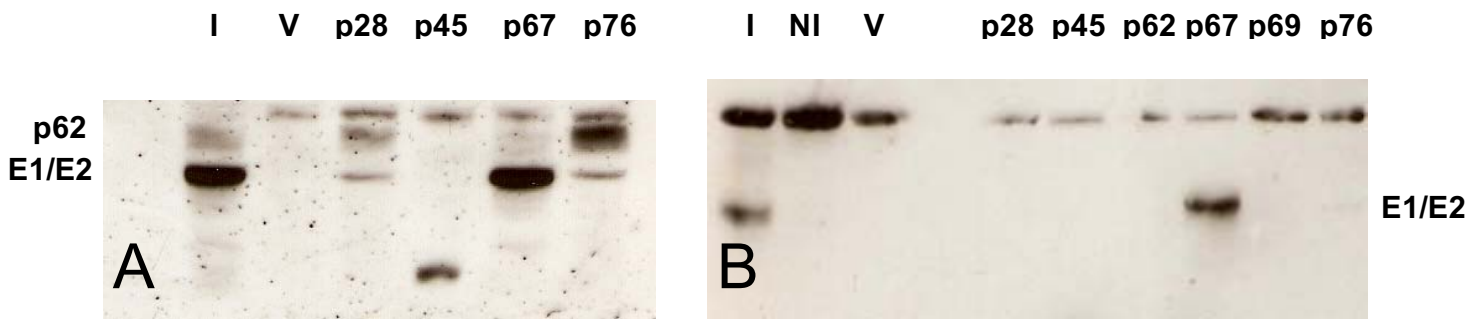

Figure 1. Western blot analysis of CHIKV proteins. Cells were infected with CHIKV (I) or transfected with the indicated recombinant vectors. Cell lysates (A) and PEG-pelleted supernatants (B) were probed with mouse anti-CHIKV polyclonal sera. The predicted viral proteins (E1, E2 and p62) are indicated. NI, non-infected cells. V, cells transfected with the pLEXm vector alone.

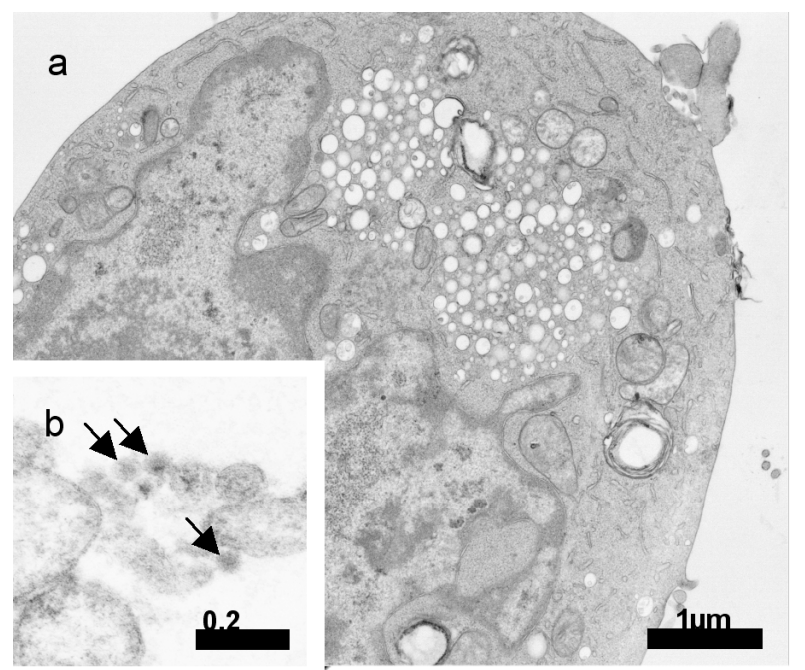

Figure 2. HEK 293T cells infected with CHIKV for $24 \mathrm{~h}$. a) Array of vesicles in the cytoplasm of the infected cells. b) Extracellular CHIKV particles (arrows)

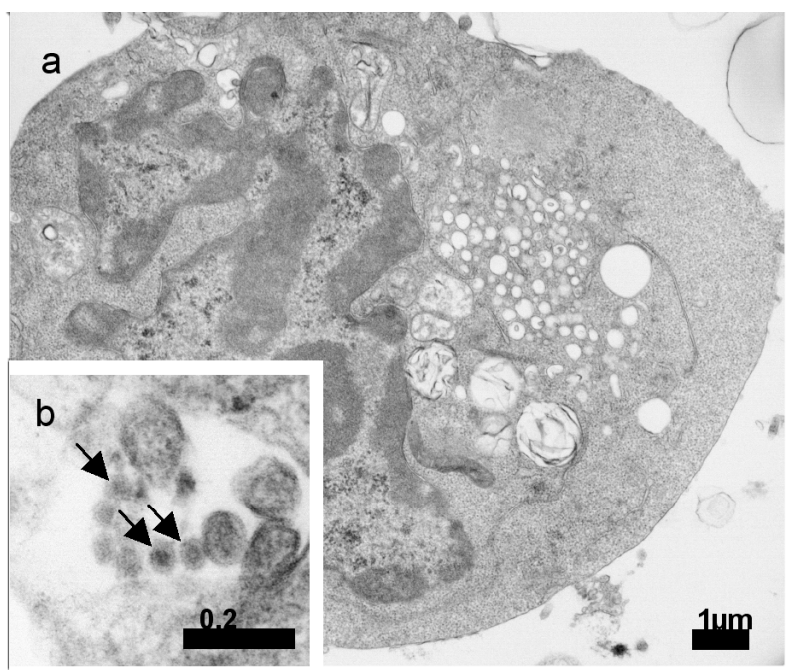

Figure 3. HEK 293T cells transfected with recombinant plasmid clone p67 for $48 \mathrm{~h}$. a) Array of vesicles in the cytoplasm of the transfected cells. b) Extracellular CHIKV VLPs (arrows). 